\title{
Virtual anatomy and pathoanatomy: Cinematic rendering of a traumatic brain injury
}

\author{
Alexander Firmötz ${ }^{1}$ and Franz A Fellner ${ }^{1,2 *}$ \\ ${ }^{1}$ Central Radiology Institute, Kepler University Hospital, Medical Faculty of the Johannes Kepler University, Linz, Austria \\ ${ }^{2}$ Medical Faculty of the Friedrich-Alexander-University of Erlangen-Nürnberg, Erlangen, Germany
}

\section{Case report}

We present a 55-year-old patient suffering a severe traumatic brain injury after falling several meters from an agricultural implement. The patient immediately lost consciousness and was brought to the emergency room. The initial polytrauma computed tomography (CT) (Figure 1) revealed a complex skull fracture, a fracture of the mandible, the zygomatic bone, and some minor lesions of the cervical spine.

Since a big fragment of the skull cap was dislocated into the orbita, the patient was moved to the neurosurgical department immediately. To provide the surgeon with additional pre-operative information, the affected area was visualized using cinematic rendering additionally (Figure 2).

The surgical procedure was performed without any complications. The bone fragments could be reduced successfully without harming any structures of the visual systems. After all the patient could be discharged in good general condition.

\section{Discussion}

While post processing techniques like multiplanar reconstructions (MPR), maximum intensity projections (MIP) or volume rendering (VR) are established since decades, cinematic rendering (CR) is a relative new technology $[1,2]$.
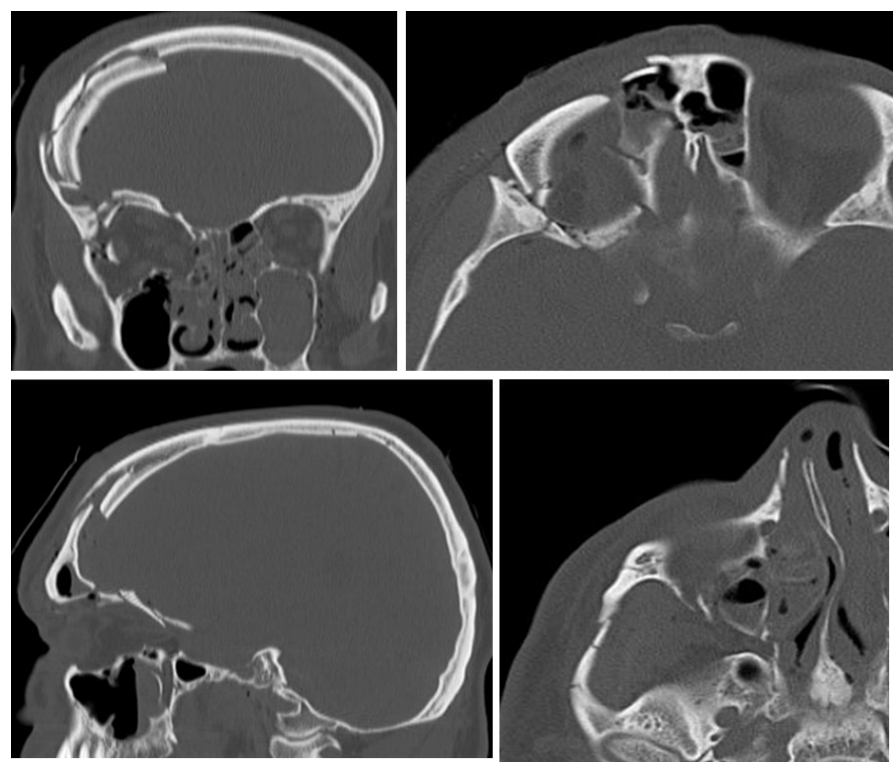

Figure 1. Initial polytrauma CT revealing a complex skull fracture

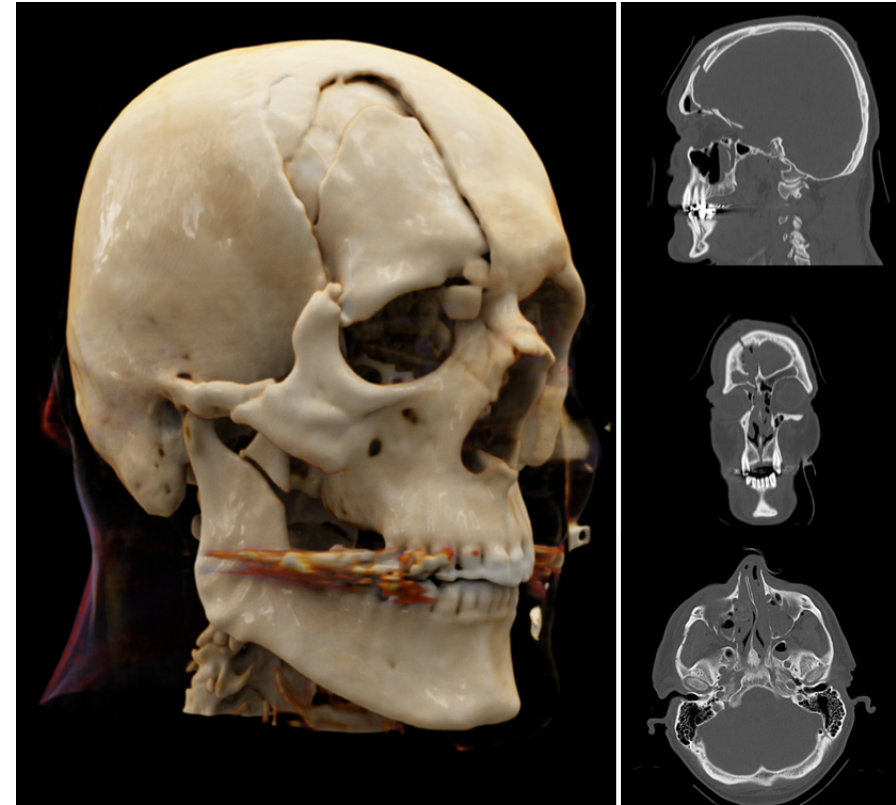

Figure 2. Three-dimensional visualization of the polytrauma CT scan using cinematic rendering

Although VR and CR are sharing the fundamentals, CR is based on an algorithm using path-tracing methods and a global illumination model to create photorealistic $3 \mathrm{D}$ images through reproducing the complex real-life physical propagation of light [3] (Figure 3). Herewith the quality of the $3 \mathrm{D}$-visualization is further enhanced by the high contrast of the bone [4]. This realistic way of showing light and shades increases the depth-discrimination for the human visual system and therefore improving the perception of those structures [5].

This case also clearly depicts the advantage of cinematic rendering in potentially enhancing pathology conspicuity (Figure 5) [6]. To get the correct impression of complex facial and/ or skull fractures as in the

${ }^{\star}$ Correspondence to: Alexander Firmötz, Central Radiology Institute, Kepler University Hospital, Medical Faculty of the Johannes Kepler University, Linz, Austria, E-mail: alexander.firmoetz@kepleruniklinikum.at

Key words: virtual anatomy, virtual pathoanatomy, cinematic rendering, computed tomography, traumatic brain injury

Received: November 18, 2020; Accepted: December 14, 2020; Published: December 17, 2020 


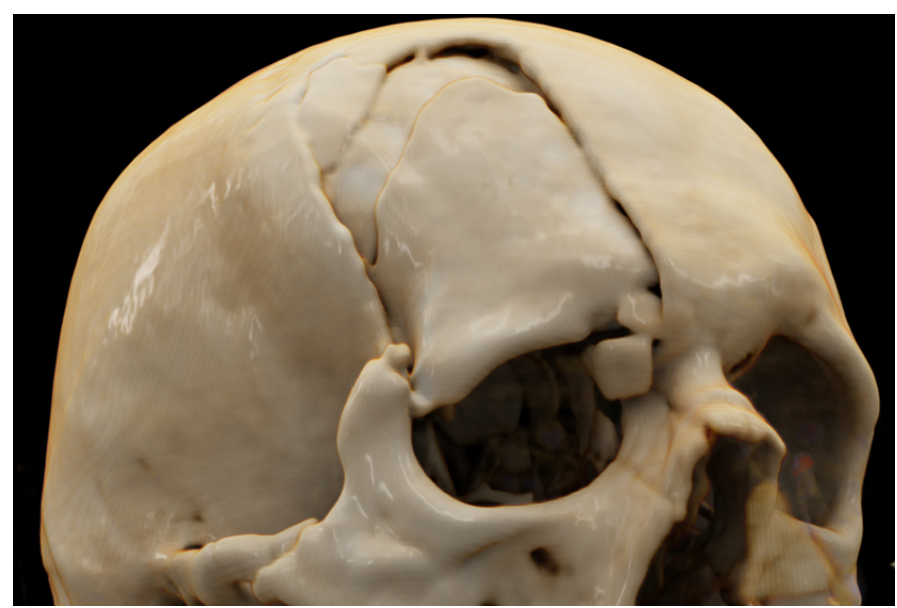

Figure 3. In cinematic rendering, the combination of path-tracing methods and a global illumination model allows a lifelike display of the affected area

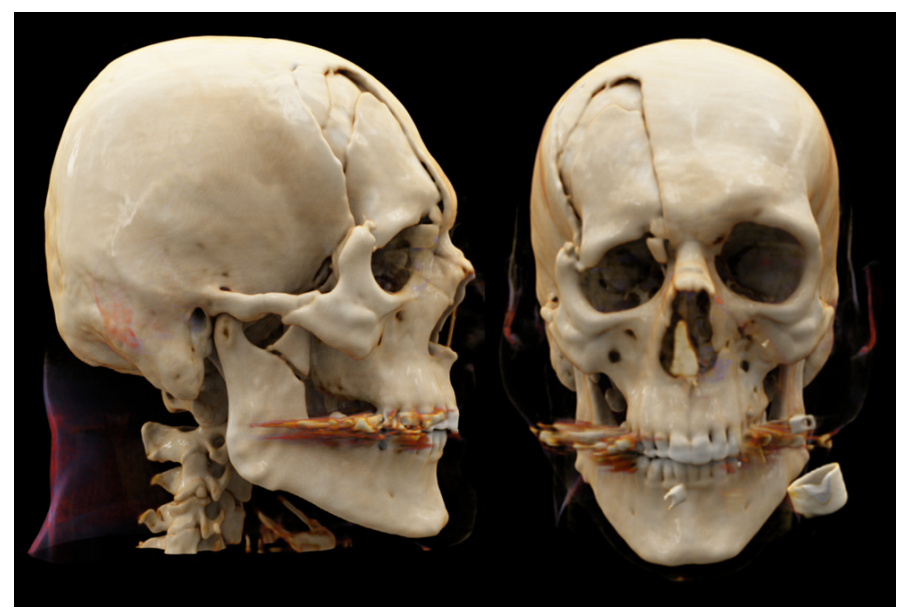

Figure 4. The combination of photorealistic display and a clear depiction of the entire anatomical region increases pathology conspicuity

case above a radiologist needs at least two different plains while a threedimensional rendering can provide that information in just one or only few pictures (Figure 4) [4,7].

Especially in trauma patients 3D-rendering techniques may offer further information about the fragment size and their relation to the surrounding structures [8]. Furthermore, the usual process of preoperative evaluation often involves the mental integration of multiple images - sometimes acquired from different modalities. By providing all those information with a single modality the time needed is reduced while the quality of the preoperative planning may be increased [9]. These advantages make cinematic rendering $3 \mathrm{D}$ visualization valuable for imaging of trauma [10-15], also in the sense of developing new methods of teaching [16-18].

\section{Disclosure}

No conflicts of interest. No grants or financial supports.

\section{References}

1. Fellner FA (2016) Introducing Cinematic Rendering: A novel technique for postprocessing medical imaging data. J Biomedical Science and Engineering 9: 170175 .

2. Dappa E, Higashigaito K, Fornaro J, Leschka S, Wildermuth S, et al. (2016) Cinematic rendering - an alternative to volume rendering for $3 \mathrm{D}$ computed tomography imaging. Insights into Imaging 7: 849-856.

3. Eid M, De Cecco CN, Nance JW Jr, Caruso D, Albrecht MH, et al. (2017) Cinematic Rendering in CT: A Novel, Lifelike 3D Visualization Technique. AJR Am J Roentgenol 209: 370-379.

4. Rowe SP, Fritz J, Fishman EK (2018) CT evaluation of musculoskeletal trauma: initial experience with cinematic rendering. Emerg Radiol 25: 93-101.

5. Langer MS, Bülthoff HH (2000) Depth discrimination from shading under diffuse lighting. Perception 2000; 29: 649-60.

6. Comaniciu D, Engel K, Georgescu B, Mansi T (2016) Shaping the future through innovations: From medical imaging to precision medicine. Med Image Anal 33: 19-26.

7. Mayer JS, Wainwright DJ, Yeakley JW, Lee KF, Harris JH Jr, et al. (1988) The role of three-dimensional computed tomography in the management of maxillofacial trauma. J Trauma 28: 1043-1053.

8. Schmidle G, Rieger M, Klauser AS, Thauerer M, Hoermann R, et al. (2014) Intraosseous rotation of the scaphoid: assessment by using a 3D CT model--an anatomic study. Eur Radiol 24: 1357-1365.

9. Elshafei M, Binder J, Baecker J, Brunner M (2019) Comparison of Cinematic Rendering and Computed Tomography for Speed and Comprehension of Surgical Anatomy. JAMA Surg 154: 738-744.

10. Fellner FA, Behawy M (2017) Cinematic and volume rendering of a scaphoid fracture. Glob Imaging Insights 2.

11. Hagleitner G, Fellner FA (2017) Ossicle vs. avulsion fracture: Cinematic rendering of MDCT data as an additional diagnostic key. Glob Imaging Insights 2.

12. Asel C, Malek M, Fellner FA (2018) Preoperative cinematic rendering of a sinus frontalis frontal bone fracture. Glob Imaging Insights 3 .

13. Firmötz A, Fellner FA (2020). Virtual anatomy and pathoanatomy: Cinematic Rendering of a proximal humerus fracture. Glob Imaging Insights 5.

14. Derler V, Fellner FA (2020) Virtual anatomy and pathoanatomy - subluxation fracture of the cervical spine. Glob Imaging Insights 5.

15. Stehrer R, Malek M, Fellner FA 2020) Cinematic Rendering for virtual anatomy and pathoanatomy in cranio-maxillofacial trauma care. Trauma Emerg Care 5.

16. Fellner FA, Berger F, Fellner C, Kremer C, Hortner H, Stocker G (2016) Volume Rendering of medical imaging data for the general public. $J$ Health Med Informat 7: 213

17. Fellner FA, Engel K, Kremer C (2017) Virtual Anatomy: The dissecting theatre of the future - implementation of Cinematic Rendering in a large $8 \mathrm{~K}$ high-resolution projection environment. J Biomedical Science and Engineering 10: 367-375.

18. Binder J, Krautz C, Engel K, Grützmann R, Fellner FA, et al. (2019) Leveraging medical imaging for medical education - a cinematic rendering-featured lecture. Ann Anat 222: 159-165.

Copyright: (C2020 Firmötz A. This is an open-access article distributed under the terms of the Creative Commons Attribution License, which permits unrestricted use, distribution, and reproduction in any medium, provided the original author and source are credited. 